\title{
Lisdexamfetamine Effects on Executive Activation and Neurochemistry in Menopausal Women with Executive Function Difficulties
}

\author{
Sheila Shanmugan ${ }^{1,2}$, James Loughead', Ravi Prakash Reddy Nanga ${ }^{3}$, Mark Elliott ${ }^{3}$, Hari Hariharan ${ }^{3}$, \\ Dina Appleby', Deborah Kim ${ }^{1,2}$, Kosha Ruparel', Ravinder Reddy ${ }^{3}$, Thomas E Brown ${ }^{4}$ and \\ C Neill Epperson*,1,2,5 \\ 'Department of Psychiatry, Perelman School of Medicine at The University of Pennsylvania, Philadelphia, PA, USA; '2Penn Center for Research on \\ Sex and Gender in Health, University of Pennsylvania, Philadelphia, PA, USA; ${ }^{3}$ Department of Radiology, Perelman School of Medicine at The \\ University of Pennsylvania, Philadelphia, PA, USA; ${ }^{4}$ Department of Psychiatry, Yale University School of Medicine, New Haven, CT, USA; \\ ${ }^{5}$ Department of Obstetrics and Gynecology, Perelman School of Medicine at The University of Pennsylvania, Philadelphia, PA, USA
}

\begin{abstract}
Many women with no history of executive dysfunction report difficulties in this domain during the menopause transition. Lisdexamfetamine (LDX) has been suggested to be a safe and effective treatment option for these women. However, the mechanism by which LDX improves executive functioning in these women is not known. Here we investigated the effects of LDX on brain activation and neurochemistry, hypothesizing that LDX would be associated with increased activation and decreased glutamate in executive regions. Fourteen women underwent multimodal neuroimaging at $7 \mathrm{~T}$ at three time points in this baseline-corrected, double-blind, placebo-controlled, crossover study. Effects of LDX on symptom severity, blood-oxygen-level-dependent (BOLD) signal, and dorsolateral prefrontal cortex (DLPFC) glutamate + glutamine (Glx) were measured using a clinician-administered questionnaire, fMRI during performance of a fractal $n$-back task, and 'H-MRS, respectively. The effect of treatment (LDX minus baseline vs placebo minus baseline) on these behavioral and neural markers of executive function was examined using repeated measures mixed effects models. LDX treatment was associated with decreased symptom severity, increased activation in the insula and DLPFC, and decreased DLPFC Glx. In addition, the magnitude of LDX-induced improvement in symptom severity predicted both direction and magnitude of LDX-induced change in insular and DLPFC activation. Moreover, symptom severity was positively correlated with Glx concentration in the left DLPFC at baseline. These findings provide novel evidence that the neural mechanisms by which LDX acts to improve self-reported executive functioning in healthy menopausal women with midlife onset of executive difficulties include modulation of insular and DLPFC recruitment as well as decrease in DLPFC Glx concentration. Neuropsychopharmacology (2017) 42, 437-445; do: I0.1038/npp.2016.162; published online I4 September 2016
\end{abstract}

\section{INTRODUCTION}

During the menopause transition, many women with no history of executive functioning deficits report cognitive difficulties in domains including working memory, organization, focus, and attention (Epperson et al, 2011). This midlife onset of executive difficulties may be a result of reduced estradiol modulation of the executive system (Shanmugan and Epperson, 2014). Proper executive functioning depends on the neurochemical environment of executive regions including the dorsolateral prefrontal cortex (DLPFC), the region of the prefrontal cortex (PFC) critical for attention and working memory (Arnsten and Jin, 2014). PFC function has

\footnotetext{
*Correspondence: Dr CN Epperson, Department of Psychiatry, Perelman School of Medicine at The University of Pennsylvania, 3535 Market Street, 3rd Floor, Philadelphia, PA 19104, USA, Tel: + I 215573 887I, Fax: + I 215573 888I, E-mail: cepp@mail.med.upenn.edu Received 3I December 20 15; revised I 5 July 20 I6; accepted 12 August 2016; accepted article preview online 23 August 2016
}

been characterized as an inverted U-shaped curve such that optimal executive function (EF) depends on a balance of factors including baseline monoaminergic and glutamatergic function (Arnsten and Jin, 2014) as well as hormonal status (Jacobs and D'Esposito, 2011). In such a model, a decrease in estradiol levels during menopause could cause executive difficulties in women whose genetics or previous life experiences make them susceptible to such a cognitive decline (Culpepper, 2015; Kudielka et al, 2009; Shanmugan and Epperson, 2014) (Figure 1).

Estradiol affects working memory through modulation of PFC dopamine activity. In women who metabolize dopamine more quickly, low estradiol levels result in improper PFC activation during working memory tasks (Jacobs and D'Esposito, 2011). Preclinical models have suggested estradiol modulation of glutamatergic function may be an additional mechanism by which estradiol loss induces changes in cognition. Estradiol loss reduces the density of healthy dendritic spines in the DLPFC (Bailey et al, 2011) 
Theoretical Model for Impact of LDX Treatment in

Menopausal Women with Executive difficulties

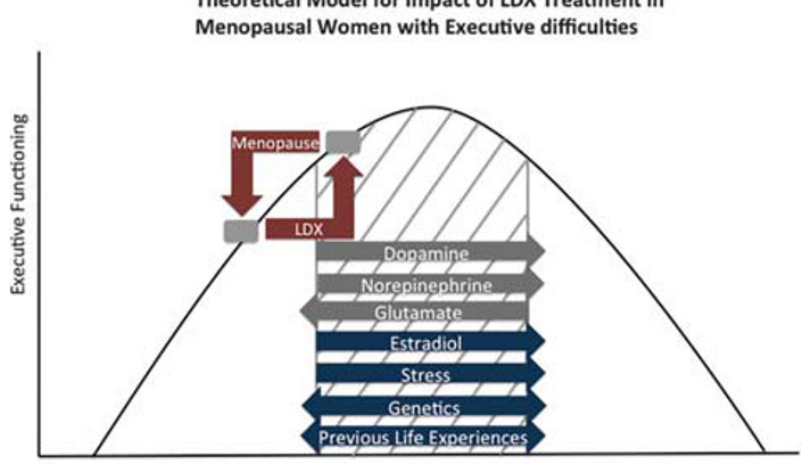

Figure I Model for effects of estradiol and LDX on executive function. Executive functioning capacity depends on the neurochemical environment in the PFC. Within the optimum range of neurotransmitter concentrations, represented by the gray crosshatching in the figure, normal executive functioning is maintained by a balance of factors including dopamine, norepinephrine, and glutamate concentrations as well as estradiol level. Where a woman falls on this curve depends on her genetics for catecholaminergic and glutamatergic metabolism and signaling as well as hormonal status and stress level. Response to stress is modified by numerous factors such as age, hormonal and reproductive status, current or previous use of medications such as glucocorticoids or psychoactive drugs, smoking, coffee and alcohol consumption, caloric intake, genetics, exposure to prenatal stress, birth weight, gestational age, level of early family adversity, and position in social hierarchy, indicating that individual lifestyle choices and adverse childhood experiences may affect an individual's executive functioning capacity before and during menopause (reviewed by Kudielka et al, 2009). In addition, factors such as depressive symptoms (Culpepper, 20 I 5), substance use, and medication side effects (Kudielka et al, 2009) may also contribute to an individual's executive functioning capacity. During menopause, women whose combination of such factors place them toward the left of the optimal range may experience executive functioning difficulties because of loss of estradiol modulation of prefrontal systems. LDX may improve executive functioning in these women by increasing dopamine and norepinephrine as well as decreasing glutamate in the PFC, although response to LDX should be considered in the context of underlying genetic variability in catecholaminergic and glutamatergic neurotransmission.

that are necessary for communication between delay cells (Wang and Arnsten, 2015), neurons capable of maintaining representations across a delay in the absence of sensory input. Loss of this regulation could lead to disinhibition of neurons that fire in response to a stimulus, potentially resulting in higher DLPFC glutamate levels and worse EF (Wang and Arnsten, 2015).

As such, interventions that increase dopamine and decrease glutamate may improve executive difficulties induced by loss of estradiol input to the PFC during menopause. We have shown that lisdexamfetamine (LDX), a stimulant medication, successfully improves subjective measures of EF in menopausal women with self-reported new-onset executive difficulties (Epperson et al, 2015). In addition to increasing extracellular dopamine and norepinephrine, stimulants also have direct and indirect effects on striatal and cortical glutamate critical to optimal PFC function (Paspalas and Goldman-Rakic, 2005).

Previous studies have shown that stimulant medications increase blood-oxygen-level-dependent (BOLD) signal in executive regions, including the insula (Rubia et al, 2014) and DLPFC (Wong and Stevens, 2012) in subjects with ADHD. In addition, stimulants also decrease glutamate levels in the PFC of children with ADHD (Wiguna et al, 2012).
However, no neuroimaging studies examining the effect of LDX on brain activation or neurochemistry have been performed. In addition, whether stimulants improve executive deficits in populations other than ADHD with similar cognitive difficulties by analogous mechanisms is not known. Furthermore, although preclinical studies suggest glutamatergic tone may mediate executive capabilities (Wang and Arnsten, 2015), information regarding the role that DLPFC glutamate levels play in EF in human subjects is limited. Thus, human subject studies evaluating the effect of stimulants on neural markers of executive dysfunction in populations other than ADHD are necessary to determine the mechanisms by which such medications improve executive deficits in these populations.

Accordingly, here we used multimodal neuroimaging and a baseline-corrected, double-blind, placebo-controlled, crossover design to examine the effects of LDX on executive system recruitment and neurochemistry in 14 healthy menopausal women with midlife-onset executive dysfunction. We hypothesized LDX would increase executive activation during a working memory task and decrease glutamate + glutamine (Glx) levels in the DLPFC at rest. Furthermore, we predicted baseline DLPFC Glx levels would be reflective of the degree of executive dysfunction.

\section{MATERIALS AND METHODS}

\section{Participants}

Subjects in this study represent a subset of women who participated in a clinical trial investigating the effects of LDX on EF (Epperson et al, 2015) and were recruited from the parent trial to participate in this neuroimaging adjunct study. Women aged 45 to 60 years with EF difficulty onset during menopause and who were within 5 years of their last menstrual period (LMP) were eligible to participate. Perimenopausal women had irregular menstrual cycles for $\geqslant 12$ months, no period for $\geqslant 3$ months, and serum folliclestimulating hormone (FSH) level of $\geqslant 20 \mathrm{IU} / \mathrm{l}$. LMP of $\geqslant 12$ months and serum FSH levels of $\geqslant 35 \mathrm{IU} / \mathrm{l}$ indicated postmenopausal status. Subjective EF symptom severity was assessed using the Brown Attention Deficit Disorder Scale (BADDS) (Brown, 1996). BADDS score of $\geqslant 20$ and onset of symptoms coinciding with the initiation of menstrual cycle irregularity were required.

Women with a lifetime history of a DSM-IV psychotic disorder or psychostimulant abuse, substance abuse disorder in the previous year, or present Axis I psychiatric disorder were excluded. Psychotropic medication use, ET use within the previous 6 months, positive pregnancy test, Mini-Mental Status Examination score of $<26$, IQ $\leqslant 90$, history of seizures, cardiac disease, active hypertension, claustrophobia, abnormal electrocardiogram at screening, left-handedness, and metallic implants were all exclusionary. Subjects were excluded from ROI analyses for poor coregistration $(n=2)$. Excessive motion (mean relative displacement $>0.5 \mathrm{~mm} ; n=1$ ) was an additional exclusionary criterion in whole-brain analyses.

\section{Study Design}

This study was a double-blind, placebo-controlled, crossover study. After screening, subjects underwent neuroimaging 
and cognitive assessment at three time points. After baseline, subjects were randomized to one pill of study medication (LDX $20 \mathrm{mg}$ or placebo) daily for the first week, two pills daily for the second week, and, if tolerated, three pills daily for the final 2 weeks of each trial. Participants were allowed to remain in the study if they could tolerate at least 1 pill per day throughout the study. Upon completion of the first 4 -week trial, participants underwent a 2 -week washout and were crossed over to the other treatment condition. Testing and imaging were conducted $\sim 2-6 \mathrm{~h}$ after last LDX dose.

\section{Assessment of Executive Function}

Assessment of subjective EF was as previously reported (Epperson et al, 2015). Briefly, subjects completed the BADDS, a validated subjective measure of EFs (Sandra Kooij et al, 2008). The BADDS is a clinician-administered questionnaire that assesses the frequency and severity of five clusters of executive dysfunction: (1) organization and activating for work, (2) sustaining attention and concentration, (3) sustaining alertness, effort, and processing speed, (4) managing affective interference, and (5) using working memory and accessing recall. Subjects rate symptoms on a scale from 0 to 3 , with 0 meaning the problem described does not relate to them and 3 indicating the problem occurs almost daily. Subjects also completed an out-of-scanner cognitive battery consisting of a letter $n$-back task, NYU paragraph recall task, and Penn Continuous Performance Task to assess working memory, verbal memory, and sustained attention, respectively (see Epperson et al, 2015 for further detail).

\section{fMRI Task Paradigm}

Subjects completed a fractal $n$-back task (Ragland et al, 2002) with 3 conditions (0-, 2-, and 3-back) during each scan to probe working memory. Subject performance was monitored to ensure adequate task engagement. See Supplementary Methods for further detail.

\section{Image Processing}

Imaging data were acquired on 7T Siemens Trio scanner (Supplementary Methods). In comparison with 3T, the higher signal-to-noise ratio and greater sensitivity to BOLD signal at 7T allows for increased ability to distinguish differences between conditions, although $7 \mathrm{~T}$ is more sensitive to distortion and artifacts.

Time series were analyzed with FEAT (fMRI Expert Analysis Tool) (Supplementary Methods). To determine the effect of treatment (active-baseline vs placebo-baseline), mean signal change for the 3-back contrast was extracted from functionally defined ROIs in the right and left DLPFC, medial frontal/cingulate gyrus (MF/CG), right and left insula, right and left parietal cortex, and posterior cingulate cortex. ROI masks for these regions were functionally defined using the parametric contrast of the group mean of all sessions (baseline, active, and placebo). The activation map of the group mean of the parametric contrast was cluster corrected at a voxel threshold of $z>4.5$ and cluster probability of $P<0.001$ (Woolrich et al, 2009). ROI masks were transformed into subject space using FLIRT. An exploratory whole-brain analysis using a two-sample paired $t$-test in FSL was also performed on the 3-back contrast to characterize the effect of treatment (active $v s$ placebo). Type I error control was provided by cluster correction using Gaussian Random Field Theory (voxel height of $z>1.6$; cluster probability of $P<0.05)$.

\section{MRS and Quantification of Glutamate}

Water-reference single voxel spectroscopy (SVS) and watersuppressed SVS scans were obtained on a voxel placed in the left DLPFC $\left(15 \times 30 \times 20 \mathrm{~mm}^{3}\right)$ (Supplementary Figure 1a). Voxels were placed $\sim 1 \mathrm{~cm}$ from the skull to avoid lipid contamination. Automated shimming of the $\mathrm{B}_{0}$ field was performed on the voxel to obtain localized water line width of $\sim 20 \mathrm{~Hz}$ or less using FASTMAP. Variable power RF pulses with optimized relaxation delays (Tkac et al, 1999) were used to obtain water suppression spectra (Cai et al, 2012). SVS for Glx was obtained using short TE SVS with modified point-resolved spectroscopy sequence (PRESS) having the following parameters: spectral width $=4 \mathrm{kHz}$, number of points $=2048$, averages $=16$ (water reference) or 64 (water suppressed), $\mathrm{TE}=20 \mathrm{~ms}, \mathrm{TR}=3000 \mathrm{~ms}$. Chemical shift artifact for Glx was minimized by setting water acquisition spectrum excitation and refocusing pulses in resonance with the water peak at 4.7 p.p.m., and also setting water-suppressed spectrum excitation and refocusing pulses in resonance with the Glx peak at 2.35 p.p.m.

Water-reference data were used to obtain channel-wise time-dependent phase shifts due to eddy current and amplitude scale factors. Using a fitting method validated by Cai et al (2012), metabolite peaks from water-suppressed spectrum were fitted as Lorentzian functions with nonlinear least squares fitting using lsqcurvefit in MATLAB. This procedure accounted for amplitudes, line widths, and peak positions for 8 macromolecular and 14 metabolite peaks. Normalization by water-reference signal allowed for quantification of Glx (Supplementary Figure 1b). See Supplementary Information for goodness-of-fit assessment.

\section{Statistical Analysis}

Mixed effects models were employed to compare the BADDS scores, cognitive task performance, BOLD in ROIs, and glutamate levels measured at the end of the active trial minus baseline $v s$ those measured at the end of the placebo minus baseline for each participant to account for repeated measures (Woolrich et al, 2009). Although the parametric activation map used to identify ROIs was corrected for multiple comparisons using Gaussian Random Field Theory (Woolrich et al, 2009), we did not further correct for the number of ROIs examined and $P$-values reported are uncorrected. In supplementary analyses, BMI was included as a covariate in models examining the effect of LDX on BOLD and glutamate to ensure LDXinduced change in BMI did not affect results. Age and the proportion of gray matter in the spectroscopy voxel were included as covariates in a model examining the drug effect on glutamate (Supplementary Results).

Spearman's rank-order correlations were used to compare (1) BADDS scores to glutamate levels at baseline and (2) change in BADDS scores between active and baseline to change in BOLD between active and baseline. Association between glutamate and BOLD was evaluated with linear 
regression. Measures of anxiety, depression, sleep, and overlap between BOLD and spectroscopy voxels were controlled for in supplementary analyses (Supplementary Methods). The effect of LDX on $n$-back performance was evaluated using mixed effects models with condition as a three-level predictor variable to minimize the effect of missing data.

\section{RESULTS}

\section{Participants}

Eighteen subjects from the parent clinical trial were enrolled in this neuroimaging adjunct study. Of these 18 subjects, 14 subjects completed all 3 imaging sessions. One subject was unable to see the screen during the fMRI task and did not complete the baseline session, two subjects withdrew after the baseline session, and one subject was unable to tolerate being in the scanner during her final scan because of nasal congestion. Baseline characteristics for the 14 women who completed both active LDX and placebo conditions are depicted in Table 1. The order of active and placebo visits across these 14 women was counterbalanced: 7 received active first and 7 received placebo first. The 14 subjects who completed imaging had higher total BADDS scores at baseline than subjects from the parent clinical trial who did not participate in this imaging adjunct study (imaging: $44.7 \pm 17.8$; other: $28.6 \pm 12.3 ; t=-3.01, \mathrm{df}=30, P=0.005)$ but were similar in terms of age, race, and education (See Supplementary Information for Methods). All but two participants finished each trial taking three pills per day. One participant experienced jitteriness and increased heart rate after increasing from one to two pills and remained on one pill for the remainder of the trial (active LDX). The other experienced an increase in blood pressure upon increasing from two to three pills and remained on two pills for the remainder of that trial (placebo).

\section{Behavioral Results}

LDX significantly decreased total BADDS $(F=26.6, d f=13$, $P<0.0002$ ) (Figure $2 \mathrm{a}$ and Supplementary Figure 2a) and each BADDS subscale (organization/activation for work, $\mathrm{F}=14.3, \mathrm{df}=13, P=0.002$; attention/concentration, $\mathrm{F}=31.7, \mathrm{df}=13, P<0.0001 ;$ alertness/effort/processing speed, $\mathrm{F}=12.3, \mathrm{df}=13, P=0.004$; managing affective interference, $\mathrm{F}=4.8, \mathrm{df}=13, P=0.05$; working memory/accessing recall, $\mathrm{F}=14.4, \mathrm{df}=13, P=0.002$ ) (Figure $2 \mathrm{~b}$ and Supplementary Figure $2 \mathrm{~b}$ ). Although there was a trend toward an effect of LDX in decreasing out-of-scanner 2-back true positive reaction time $(\mathrm{F}=3.2, \mathrm{df}=10, P=0.1)$, no significant effect of LDX was observed on delayed paragraph recall, continuous performance task, or in-scanner $n$-back performance $(P>0.05)$ (Supplementary Figure 3).

\section{BOLD Results}

As expected, the $n$-back task robustly activated executive network regions and deactivated nonexecutive regions (Figure 3a). ROI analyses demonstrated LDX increased activation in the right insula/IFG $(\mathrm{F}=20.7, \mathrm{df}=11$, $P=0.0008)$ and left DLPFC $(\mathrm{F}=2.9, \mathrm{df}=11, P=0.12)$ (Figure $3 \mathrm{~b}$ and Supplementary Table 1) during the 3-back.
Table I Participant Characteristics

Mean (SD) or number (\%)

\begin{tabular}{|c|c|}
\hline Age (years) & $53.1(3.0)$ \\
\hline Time since last menstrual period (months) & $31.8(\mid 8.1)$ \\
\hline IQ & $115.9(\mid 1.3)$ \\
\hline Mini-mental status exam & $28.8(1.1)$ \\
\hline \multicolumn{2}{|l|}{ Marital status } \\
\hline Single & $2(14.3)$ \\
\hline Married & $9(64.3)$ \\
\hline Divorced/separated & $2(14.3)$ \\
\hline Widowed & $0(0)$ \\
\hline Did not disclose & । (7.1) \\
\hline \multicolumn{2}{|l|}{ Menopause status } \\
\hline Perimenopause & $2(14.3)$ \\
\hline Postmenopause & $12(85.7)$ \\
\hline \multicolumn{2}{|l|}{ Race } \\
\hline Caucasian & $9(64.3)$ \\
\hline African American & $3(21.4)$ \\
\hline American Indian/Alaska native & । (7.I) \\
\hline Others & । (7.1) \\
\hline \multicolumn{2}{|l|}{ Ethnicity } \\
\hline Hispanic & $0(0)$ \\
\hline Non-Hispanic & $14(100)$ \\
\hline \multicolumn{2}{|l|}{ Education } \\
\hline High school & । (7.1) \\
\hline Some college/vocational & $2(14.3)$ \\
\hline College graduate/some graduate school & $6(42.9)$ \\
\hline Graduate/professional degree & $5(35.7)$ \\
\hline \multicolumn{2}{|l|}{ Household income } \\
\hline Unknown or did not disclose & $4(28.6)$ \\
\hline$<\$ 50000$ & $2(14.3)$ \\
\hline$\$ 50000$ to $\$ 100000$ & $3(21.4)$ \\
\hline$\$ 100000$ to $\$ 200000$ & $4(28.6)$ \\
\hline$>\$ 200000$ & $0(0)$ \\
\hline \multicolumn{2}{|l|}{ Employed } \\
\hline Full time & $14(100)$ \\
\hline
\end{tabular}

This robust insular finding remained significant in an exploratory whole-brain analysis (MNI coordinates: $x=48$, $y=52, z=18 ; k=1416$ ) (Figure 3c).

LDX-induced change in right insula activation was positively associated with LDX-induced decrease in total BADDS score $(r=0.95, P=<0.0001, t=9.88, \mathrm{df}=11)$, BADDS subscale $1(r=0.86, P=0.0004, t=5.22, \mathrm{df}=11)$, and BADDS subscale $3(r=0.83, P=0.0008, t=4.70, \mathrm{df}=$ 11). Similar results were obtained for correlation between LDX-induced change in activation in the left DLPFC and total BADDS score $(r=0.78, P=0.003, t=3.90, \mathrm{df}=11)$, BADDS subscale $1(r=0.78, P=0.0028, t=3.93, \mathrm{df}=11)$, and BADDS subscale $3(r=0.81, P=0.0014, t=4.38, \mathrm{df}=11)$ (Figure $3 \mathrm{~d}$ ). Correlations remained significant when controlling for measures of anxiety, depression, and sleep (Supplementary Results). 
a

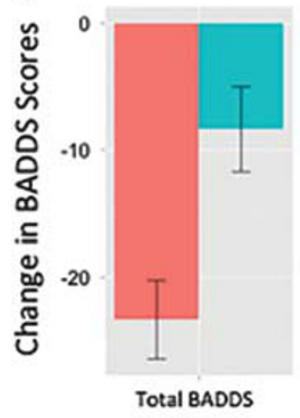

b

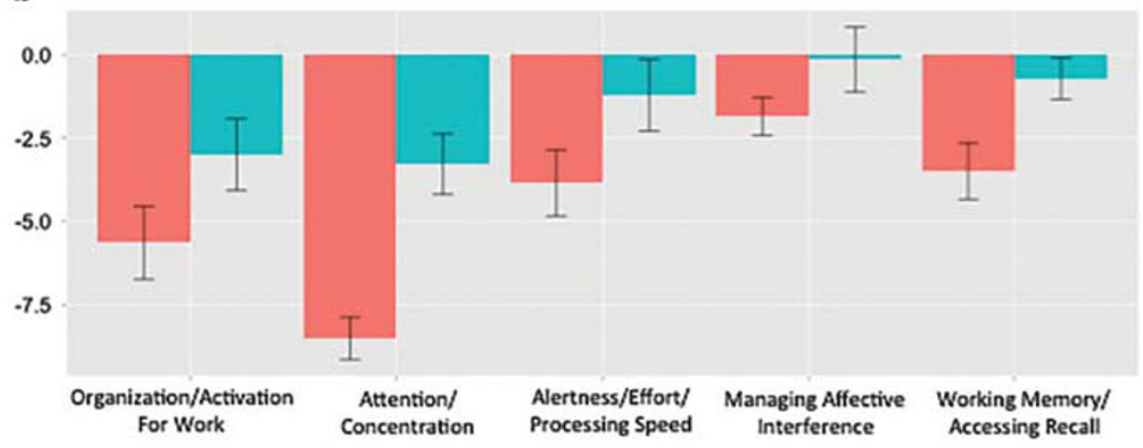

Active - Baseline

Placebo - Baseline

Figure 2 LDX improves subjective measures of executive function. The decrease in BADDS scores from baseline was significantly greater with LDX versus placebo for both (a) total BADDS $(F=26.6, \mathrm{df}=13, P<0.0002)$ and (b) each BADDS subscale (organization/activation for work, $F=|4.3, \mathrm{df}=| 3, P=0.002$; attention/concentration, $F=31.7, \mathrm{df}=13, P<0.0001$; alertness/effort/processing speed, $=12.3, \mathrm{df}=13, P=0.004$; managing affective interference, $F=4.8$, $\mathrm{df}=13, P=0.05$; working memory/accessing recall, $\mathrm{F}=14.4, \mathrm{df}=13, P=0.002$ ). Error bars represent standard error.

a
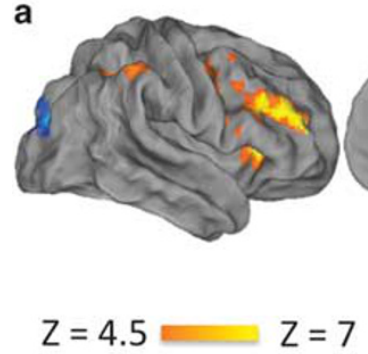

$Z=1.9$

$Z=6$

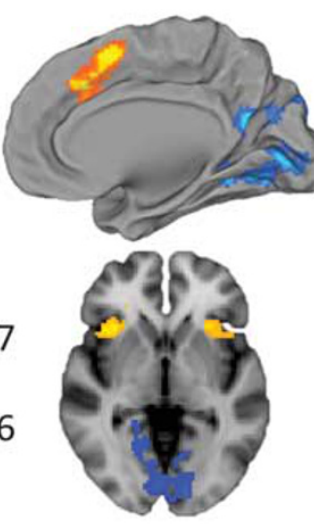

C

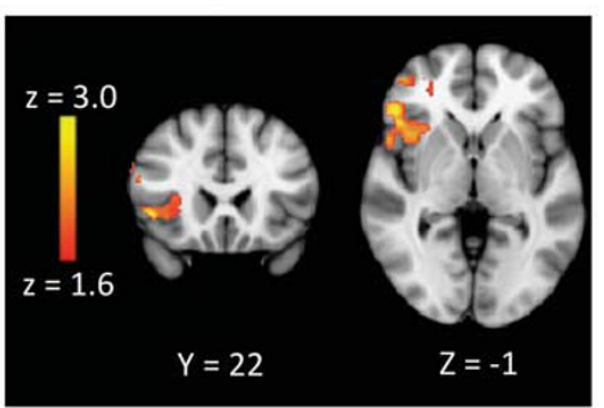

b
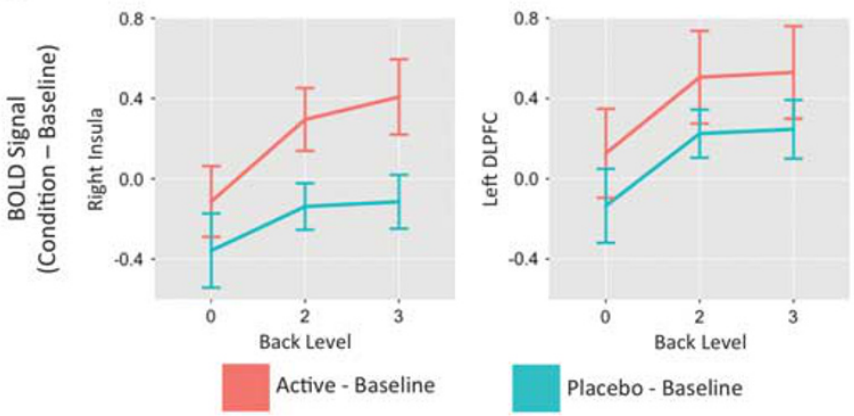

Placebo - Baseline d

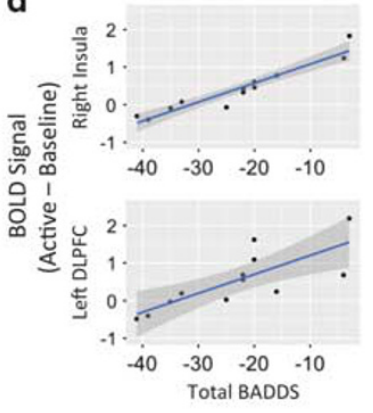

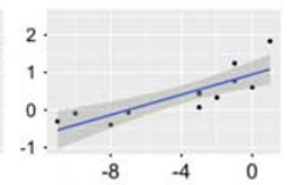

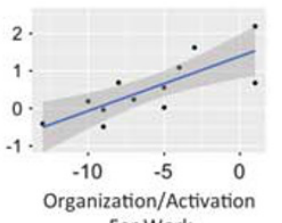

For Work

BADDS Score (Active - Baseline)

Figure 3 LDX increases executive activation during working memory. (a) The n-back task robustly activated executive network regions and deactivated non-executive regions, as depicted by the parametric contrast $(z>4.5, P<0.00 \mathrm{I})$. (b) Recruitment of the right insula and left DLPFC increased with increasing levels of working memory load. Values plotted represent mean difference in activation from baseline at each task level. Error bars represent SD. (c) Wholebrain analysis using a paired t-test $(z>1.6, P<0.05)$ of the 3-back contrast demonstrated that activation in the right insula (MNI coordinates: $x=48, y=52$, $z=\mid 8 ; k=1416$ ) was significantly greater than placebo. (d) LDX-induced increase in both right insula and left DLPFC BOLD signal from baseline during the 3-back was positively correlated with LDX-induced decrease in total BADDS Scores as well as BADDS subscales measuring organization/activation for work and alertness/effort/processing speed. Subjects with the greatest improvements in BADDS scores demonstrated less task-induced activation with LDX, whereas subjects with the least improvements in BADDS scores demonstrated greater task-induced activation with LDX. Right insula: total BADDS $(r=0.95$, $P \leqslant 0.000 \mathrm{I}, t=9.88, \mathrm{df}=1 \mathrm{I})$; organization/activation for work $(r=0.86, P=0.0004, t=5.22, \mathrm{df}=1 \mathrm{I})$; alertness/effort/processing speed $(r=0.83, P=0.0008$, $t=4.70, \mathrm{df}=1 \mathrm{I})$. Left DLPFC: total BADDS $(r=0.78, P=0.003, t=3.90, \mathrm{df}=1 \mathrm{I})$; organization/activation for work $(r=0.78, P=0.0028, t=3.93, \mathrm{df}=1 \mathrm{I})$; alertness/effort/processing speed $(r=0.81, P=0.0014, t=4.38, \mathrm{df}=1 \mathrm{I})$. 

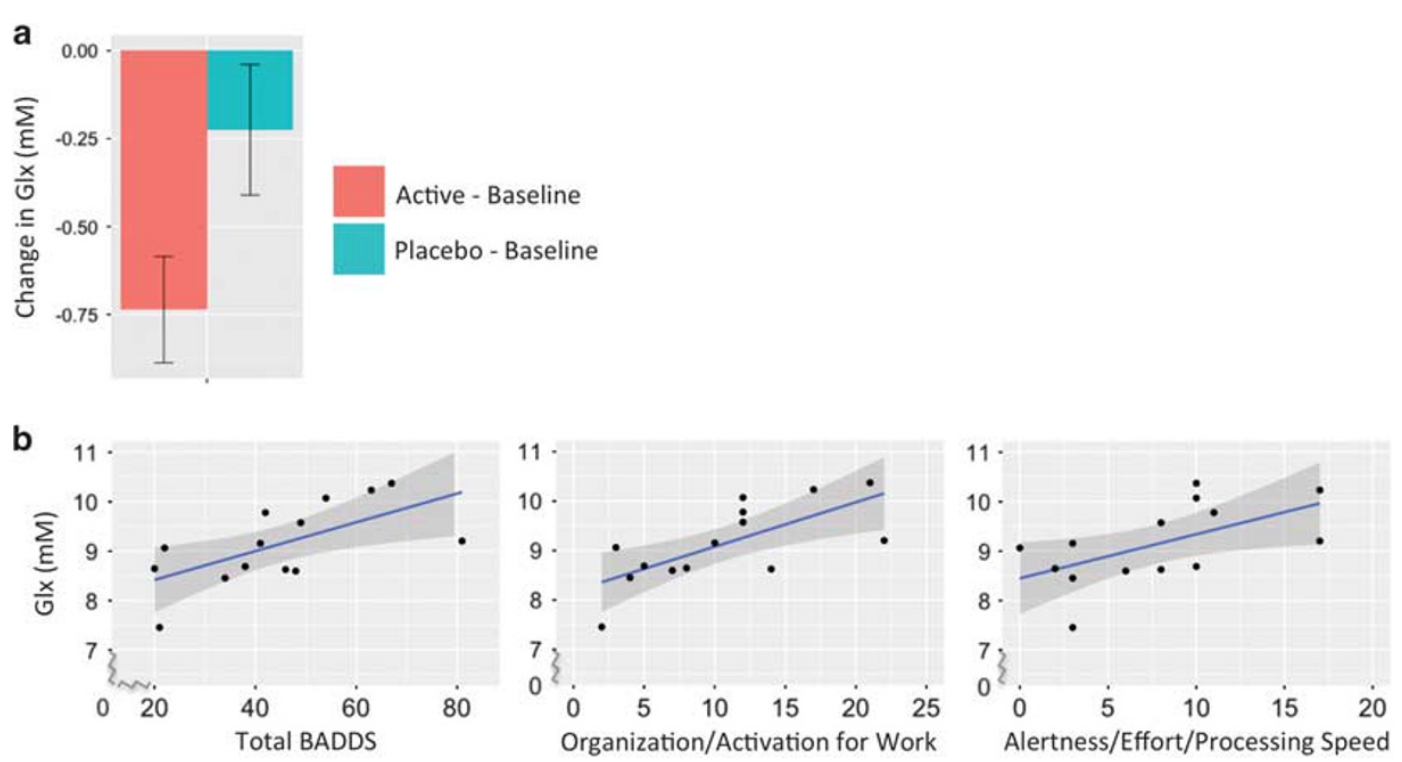

Figure 4 Left DLPFC Glx concentrations. (a) Decrease in left DLPFC Glx concentration from baseline was greater with $L D X$ vs placebo $(F=4.5$, $d f=\mid 2$, $P=0.056)$. Error bars represent standard error. (b) At baseline, left DLPFC Glx concentration was positively correlated with total BADDS $(r=0.64, P=0.01$, $t=2.90, \mathrm{df}=13)$ as well as BADDS subscales measuring organization/activation for work $(r=0.70, P=0.005, t=3.43, \mathrm{df}=13)$ and alertness/effort/processing speed $(r=0.58, P=0.03, t=2.47, d f=13)$.

\section{Spectroscopy Results}

LDX was associated with decreased Glx concentration in the left DLPFC $(\mathrm{F}=4.6, \mathrm{df}=13, P=0.052)$ (Figure $4 \mathrm{a}$ and Supplementary Figure 4). Glx values (mean $\mathrm{mM} \pm \mathrm{SD}$ ) at baseline, active, and placebo were $9.14 \pm 0.81,8.4 \pm 1.03$, and $8.91 \pm 0.86$, respectively. At baseline, left DLPFC Glx concentration was positively correlated with total BADDS $(r=0.64, P=0.01, t=2.90, \mathrm{df}=13)$, BADDS subscale 1 $(r=0.70, P=0.005, t=3.43, \mathrm{df}=13)$, and BADDS subscale $3(r=0.58, P=0.03, t=2.47, \mathrm{df}=13)$ (Figure $4 \mathrm{~b})$. There were no other significant correlations between Glx and BADDS or between Glx and DLPFC BOLD $(P>0.05)$.

\section{DISCUSSION}

In this double-blind, placebo-controlled, crossover study, we examined the effect of LDX on behavioral and neural markers of EF at 7T. To account for the inherent variability in the BOLD signal, a repeated measures, baseline-corrected study design was used. LDX treatment was associated with improved self-reported $\mathrm{EF}$ in comparison with placebo, as shown by a significant reduction in BADDS scores. Furthermore, LDX treatment significantly increased recruitment of executive regions during performance of a working memory task. In addition, LDX treatment decreased Glx concentrations in the left DLPFC at rest. Taken together, these data provide novel evidence for the neural mechanisms by which this drug acts to improve self-reported EF in previously healthy menopausal women with midlife onset of executive difficulties.

\section{Evidence for Psychostimulant Effects on EF}

As in the parent clinical trial (Epperson et al, 2015), we found that LDX improved EF in this subset of women as measured by self-reported symptom severity. We did not, however, find an effect of LDX on working memory task performance. Although the task used in this study was validated in a large sample of healthy volunteers (Gur et al, 2010), education level has been shown to be correlated with performance on executive tasks, particularly the $n$-back (Gur et al, 2010). Given the large portion of our sample that had at least a college degree (79\%) and graduate degree (35\%), ceiling effects from the high performance at baseline likely rendered these tests unable to detect any objective improvements in EF.

\section{Evidence for Psychostimulant Effects on Executive Activation}

The most robust finding in the present study is evidence of LDX effect of increasing activation in the insula/IFG region. This was observed in an ROI analysis at all levels of the $n$-back task performed as well as in a whole-brain analysis. These results accord with a copious literature of neuroimaging studies of stimulant effects in individuals with ADHD (Rubia et al, 2014), and moreover highlight the mechanism of this drug's action in a population who exhibits similar executive deficits but who do not meet criteria for ADHD.

A meta-analysis of $14 \mathrm{fMRI}$ data sets found stimulants relative to placebo/off-medication most consistently increase activation in the insula/IFC (Rubia et al, 2014). This finding is present during tasks probing executive domains of interference inhibition (Rubia et al, 2014), time discrimination (Rubia et al, 2014), and working memory (Spencer et al, 2013). The insula is part of the cinglo-opercular control network critical for cognitive control, and is particularly important in the maintenance of task sets and error monitoring (Power et al, 2011).

Although the DLPFC is a part of the frontoparietal network supporting goal-directed executive processes (Cortese et al, 2012), and is an area implicated in the pathogenesis of executive deficits seen in ADHD (Cortese et al, 2012), the 
effect of stimulants on the DLPFC is inconsistent. Studies have found that stimulants increase (Wong and Stevens, 2012), decrease (Cubillo et al, 2014), or have no effect (Kobel et al, 2009; Rubia et al, 2014) on DLPFC activation. Such inconsistency may be due in part to the type or dosage of medication used and level of working memory load probed (Cubillo et al, 2014).

We also found the magnitude of LDX-induced improvement in symptom severity predicted both the direction and magnitude of LDX-induced change in both insular and DLPFC activation. Whereas subjects with the greatest improvements in BADDS scores demonstrated less taskinduced activation, subjects with the least improvements in BADDS scores demonstrated greater task-induced activation, suggesting that other factors such as genetics, adverse childhood experiences, baseline activation and symptom severity, and time since last menstrual period affect the relationship between LDX treatment and brain and behavioral response. Our observation that LDX treatment resulted in an overall increase in DLPFC and insular cortex BOLD (Figure $3 b$ and c) was likely driven by the greater proportion of individuals who experienced an increase rather than a decrease in activation from baseline (Figure 3d). One study found no correlation between activation during a stopsignal task and symptom severity after controlling for psychostimulant use in adults with ADHD (Congdon et al, 2014). However, no studies have looked at the correlation between stimulant-induced changes in brain activation during working memory and stimulant-induced changes in symptom severity in adults. Thus, our findings make an important step toward understanding factors that affect stimulant effects on brain activation and may in part explain the contradictory findings (Cubillo et al, 2014; Kobel et al, 2009; Rubia et al, 2014; Wong and Stevens, 2012) of stimulants on brain activation in the literature.

\section{Evidence for Psychostimulant Effects on Neurochemistry}

A third important finding in this study was that LDX significantly decreases Glx concentrations in the DLPFC from baseline in comparison with placebo. Results of the few studies that have examined the effect of stimulants on left PFC glutamate levels are inconsistent and have shown that stimulants either decrease (Wiguna et al, 2012) or have no effect (Carrey et al, 2002; Husarova et al, 2014b) on glutamate concentrations. Differential effects of stimulants on glutamate levels have been attributed to differences in medication formulation, treatment duration, or a combination of both (Husarova et al, 2014b).

Importantly, we found that left DLPFC Glx levels at baseline positively correlated with self-reported executive function symptom severity. Only one other study has examined the relationship between DLPFC glutamate concentration and subjective executive dysfunction symptom severity (Husarova et al, 2014a). Although this study found no correlation between left DLPFC glutamate and parentreported symptom severity, associations between executive symptom severity and other neurometabolites including $\mathrm{N}$-acetylaspartate/creatine, Glx/creatine, and choline/creatine in the DLPFC or white matter behind the DLPFC were observed. However, it is important to note that subjects in that study were children who met criteria for ADHD combined subtype and almost one-third of subjects also had comorbid oppositional defiant disorder.

Interestingly, we did not find an association between treatment-induced change in Glx and treatment-induced change in symptom severity. This would seem to indicate that LDX disrupts the relationship between Glx and symptom severity seen at baseline. The implications of the lack of association between these variables with placebo treatment is less clear, but could indicate that either placebo also causes a change in neurochemistry, prior LDX treatment alters the relationship between Glx and symptom severity, or that the washout period was not of sufficient duration to return the relationship between these variables to baseline. Glutamate antagonists have been shown to improve symptoms of anxiety and depression in preclinical models (Gerhard et al, 2016), suggesting the moderating effect of glutamate on mood symptoms could be a confounding factor when evaluating the relationship between glutamate and executive symptom severity. However, we found that correlations between Glx and total BADDS score remained significant even when controlling for measures of depression and sleep, indicating that this relationship is not simply a reflection of the effect of LDX on mood. No other studies have directly examined the correlation between stimulant-induced changes in left DLPFC Glx and stimulant-induced changes in symptom severity.

We did not find an association between Glx concentration and BOLD signal in the left DLPFC. Interestingly, both of these measures were correlated with the same measures of symptom severity: total BADDS, subscale 1 , and subscale 3 . There was not, however, an association between neural outcome measures and other BADDS subscales even though LDX significantly improved symptoms in all domains, suggesting certain subscales may be more sensitive to neural markers of EF in this population. The relationship between glutamate concentration and BOLD signal is not well established. One study found a positive correlation between dorsal anterior cingulate cortex (ACC) glutamate and BOLD during a cognitive control task in several regions including the retrosplenial cortex, orbitofrontal cortex, inferior parietal lobule, and basal ganglia (Falkenberg et al, 2012). Another study found a positive correlation between dorsomedial PFC glutamate and pregenual ACC BOLD during emotional processing (Stan et al, 2014). In contrast, another study found no correlation between glutamate and BOLD in the ACC or inferior frontal gyrus before, during, or after performance of an interference inhibition task (Kuhn et al, 2015), even though voxel placement was guided by individual's BOLD activity. However, no studies have examined the relationship between DLPFC glutamate concentration and activation during a working memory task at baseline or with psychostimulant treatment.

\section{Limitations}

Certain limitations of the present study should be noted. First, the sample size is relatively small. However, the repeated measures design allowed for decreased variance in estimates of treatment effects and increased power to detect between group differences. In addition, although the BOLD signal is inherently variable, this study included a baseline session allowing comparisons between active and placebo to be baseline corrected. Doing so contrasted the minimal 
differences in behavior, executive activation, and Glx concentrations between placebo and baseline with the significant differences between active and baseline. Furthermore, the high signal-to-noise ratio at $7 \mathrm{~T}$ allows for increased ability to distinguish differences between conditions.

A second limitation of this study is that ${ }^{1} \mathrm{H}$-MRS does not distinguish between intra- and extracellular glutamate levels. Preclinical studies show the extracellular pool of glutamate is between 1 and $4 \mu \mathrm{mol}$ (Lerma et al, 1986). Glutamate measured using ${ }^{1} \mathrm{H}$-MRS is between 8 and $12 \mathrm{mmol}$, suggesting our findings are based primarily on the intracellular pool of glutamate. Another limitation is the inability to resolve glutamate from glutamine, even at $7 \mathrm{~T}$, because of spin-spin coupling. However, the small values in Glx fit uncertainty $(<0.1)$ and strong intraclass correlation (0.6) between Glx values at baseline and placebo suggest robust goodness of fit and test-retest reliability, respectively.

Third, current substance abuse could affect both behavioral and imaging outcomes and subjects were not required to undergo a drug screen on each test day. However, a clear urine toxicology during screening was required for inclusion in the study. In addition, because these subjects did not have any history of substance abuse, substance abuse during the study was unlikely. Fourth, we did not formally assess whether participants could accurately guess when they were on active LDX or placebo. However, we did examine carryover effects in the larger clinical trial and did not find any order effects on behavioral outcome measures (Epperson et al, 2015), suggesting that if participants accurately identified the active LDX from placebo trials, this knowledge did not unduly affect our outcomes.

\section{CONCLUSIONS}

In summary, these data provide novel evidence regarding the neural mechanisms by which stimulants act to improve executive functioning in healthy menopausal women with midlife onset of executive difficulties. Further research is needed to determine whether other factors such as genetics or adverse childhood experiences modulate the effect of LDX on glutamate and executive system recruitment. Such information could serve as markers of women who may experience cognitive difficulties upon entering menopause and of women who would benefit from early intervention. Furthermore, the mechanisms by which loss of estradiol and healthy dendritic spines as well as higher cortical glutamate concentrations contribute to worse executive functioning in human subjects are yet to be elucidated, and future research should consider the role of such factors in promoting neuroinflammation and oxidative stress (Kitamura et al, 2009). Additional research in a larger sample would be helpful in ascertaining whether there is a three-way interaction between symptom severity, BOLD, and Glx. A larger sample may also reveal an effect of menopause stage on these measures. Executive function has been shown to decline between early and late postmenopause (Elsabagh et al, 2007), although declines observed in other cognitive domains such as verbal memory (Epperson et al, 2013) suggest that executive dysfunction may begin earlier. Moreover, it would be important to confirm whether LDX acts by similar mechanisms after surgery- or chemotherapy-induced menopause.

\section{FUNDING AND DISCLOSURE}

Dr Epperson discloses personal investments in the following companies; Pfizer, Johnson and Johnson, Merck, Abbott, and Abbvie. Dr Brown reports consultation fees from Ironshore Pharmaceuticals and Lundbeck Pharmaceuticals. He also receives publication royalties from American Psychiatric Publishing, Jossey-Bass/Wiley, Psychological Corporation/ Pearson, Routledge, and Yale University Press. The other authors declare no conflict of interest.

\section{ACKNOWLEDGMENTS}

Dr Epperson and Dr Brown received funding from Shire Pharmaceuticals for investigator-initiated research.

\section{REFERENCES}

Arnsten AF, Jin LE (2014). Molecular influences on working memory circuits in dorsolateral prefrontal cortex. Prog Mol Biol Transl Sci 122: 211-231.

Bailey ME, Wang AC, Hao J, Janssen WG, Hara Y, Dumitriu D et al (2011). Interactive effects of age and estrogen on cortical neurons: implications for cognitive aging. Neuroscience 191: 148-158.

Brown TE (1996). Brown Attention Deficit Disorder Scales for Adolescents and Adults. The Psychological Corporation: San Antonio, TX.

Cai K, Nanga RP, Lamprou L, Schinstine C, Elliott M, Hariharan H et al (2012). The impact of gabapentin administration on brain GABA and glutamate concentrations: a 7T (1)H-MRS study. Neuropsychopharmacology 37: 2764-2771.

Carrey N, MacMaster FP, Sparkes SJ, Khan SC, Kusumakar V (2002). Glutamatergic changes with treatment in attention deficit hyperactivity disorder: a preliminary case series. J Child Adolesc Psychopharmacol 12: 331-336.

Congdon E, Altshuler LL, Mumford JA, Karlsgodt KH, Sabb FW, Ventura J et al (2014). Neural activation during response inhibition in adult attention-deficit/hyperactivity disorder: preliminary findings on the effects of medication and symptom severity. Psychiatry Res 222: 17-28.

Cortese S, Kelly C, Chabernaud C, Proal E, Di Martino A, Milham MP et al (2012). Toward systems neuroscience of ADHD: a metaanalysis of $55 \mathrm{fMRI}$ studies. Am J Psychiatry 169: 1038-1055.

Cubillo A, Smith AB, Barrett N, Giampietro V, Brammer M, Simmons A et al (2014). Drug-specific laterality effects on frontal lobe activation of atomoxetine and methylphenidate in attention deficit hyperactivity disorder boys during working memory. Psychol Med 44: 633-646.

Culpepper L (2015). Impact of untreated major depressive disorder on cognition and daily function. J Clin Psychiatry 76: e901.

Elsabagh S, Hartley DE, File SE (2007). Cognitive function in late versus early postmenopausal stage. Maturitas 56: 84-93.

Epperson CN, Pittman B, Czarkowski KA, Bradley J, Quinlan DM, Brown TE (2011). Impact of atomoxetine on subjective attention and memory difficulties in perimenopausal and postmenopausal women. Menopause 18: 542-548.

Epperson CN, Sammel MD, Freeman EW (2013). Menopause effects on verbal memory: findings from a longitudinal community cohort. J Clin Endocrinol Metabol 98: 3829-3838.

Epperson CN, Shanmugan S, Kim DR, Mathews S, Czarkowski KA, Bradley J et al (2015). New onset executive function difficulties at 
menopause: a possible role for lisdexamfetamine. Psychopharmacology 232: 3091-3100.

Falkenberg LE, Westerhausen R, Specht K, Hugdahl K (2012). Resting-state glutamate level in the anterior cingulate predicts blood-oxygen level-dependent response to cognitive control. Proc Natl Acad Sci USA 109: 5069-5073.

Gerhard DM, Wohleb ES, Duman RS (2016). Emerging treatment mechanisms for depression: focus on glutamate and synaptic plasticity. Drug Discov Today 21: 454-464.

Gur RC, Richard J, Hughett P, Calkins ME, Macy L, Bilker WB et al (2010). A cognitive neuroscience-based computerized battery for efficient measurement of individual differences: standardization and initial construct validation. J Neurosci Methods 187: 254-262.

Husarova V, Bittsansky M, Ondrejka I, Dobrota D (2014a). Correlations of ADHD symptoms with neurometabolites measured by $1 \mathrm{H}$ magnetic resonance spectroscopy. Bratisl Lek Listy 115: 635-642.

Husarova V, Bittsansky M, Ondrejka I, Dobrota D (2014b). Prefrontal grey and white matter neurometabolite changes after atomoxetine and methylphenidate in children with attention deficit/hyperactivity disorder: a (1) $\mathrm{H}$ magnetic resonance spectroscopy study. Psychiatry Res 222: 75-83.

Jacobs E, D'Esposito M (2011). Estrogen shapes dopaminedependent cognitive processes: implications for women's health. J Neurosci 31: 5286-5293.

Kitamura N, Araya R, Kudoh M, Kishida H, Kimura T, Murayama $\mathrm{M}$ et al (2009). Beneficial effects of estrogen in a mouse model of cerebrovascular insufficiency. PLoS One 4: e5159.

Kobel M, Bechtel N, Weber P, Specht K, Klarhofer M, Scheffler K et al (2009). Effects of methylphenidate on working memory functioning in children with attention deficit/hyperactivity disorder. Eur J Paediatr Neurol 13: 516-523.

Kudielka BM, Hellhammer DH, Wust S (2009). Why do we respond so differently? Reviewing determinants of human salivary cortisol responses to challenge. Psychoneuroendocrinology 34: 2-18.

Kuhn S, Schubert F, Mekle R, Wenger E, Ittermann B, Lindenberger $U$ et al (2015). Neurotransmitter changes during interference task in anterior cingulate cortex: evidence from fMRIguided functional MRS at 3T. Brain Struct Funct 221: 2541-2551.

Lerma J, Herranz AS, Herreras O, Abraira V, Martin del Rio R (1986). In vivo determination of extracellular concentration of amino acids in the rat hippocampus. A method based on brain dialysis and computerized analysis. Brain Res 384: 145-155.

Paspalas CD, Goldman-Rakic PS (2005). Presynaptic D1 dopamine receptors in primate prefrontal cortex: target-specific expression in the glutamatergic synapse. J Neurosci 25: 1260-1267.
Power JD, Cohen AL, Nelson SM, Wig GS, Barnes KA, Church JA et al (2011). Functional network organization of the human brain. Neuron 72: 665-678.

Ragland JD, Turetsky BI, Gur RC, Gunning-Dixon F, Turner T, Schroeder L et al (2002). Working memory for complex figures: an fMRI comparison of letter and fractal n-back tasks. Neuropsychology 16: 370-379.

Rubia K, Alegria AA, Cubillo AI, Smith AB, Brammer MJ, Radua J (2014). Effects of stimulants on brain function in attentiondeficit/hyperactivity disorder: a systematic review and metaanalysis. Biol Psychiatry 76: 616-628.

Sandra Kooij JJ, Marije Boonstra A, Swinkels SH, Bekker EM, de Noord I, Buitelaar JK (2008). Reliability, validity, and utility of instruments for self-report and informant report concerning symptoms of ADHD in adult patients. J Atten Disord 11: 445-458.

Shanmugan S, Epperson CN (2014). Estrogen and the prefrontal cortex: towards a new understanding of estrogen's effects on executive functions in the menopause transition. Hum Brain Mapp 35: 847-865.

Spencer TJ, Brown A, Seidman LJ, Valera EM, Makris N, Lomedico A et al (2013). Effect of psychostimulants on brain structure and function in ADHD: a qualitative literature review of magnetic resonance imaging-based neuroimaging studies. J Clin Psychiatry 74: 902-917.

Stan AD, Schirda CV, Bertocci MA, Bebko GM, Kronhaus DM, Aslam HA et al (2014). Glutamate and GABA contributions to medial prefrontal cortical activity to emotion: implications for mood disorders. Psychiatry Res 223: 253-260.

Tkac I, Starcuk Z, Choi IY, Gruetter R (1999). In vivo 1H NMR spectroscopy of rat brain at $1 \mathrm{~ms}$ echo time. Magn Reson Med 41: 649-656.

Wang M, Arnsten AF (2015). Contribution of NMDA receptors to dorsolateral prefrontal cortical networks in primates. Neurosci Bull 31: 191-197.

Wiguna T, Guerrero AP, Wibisono S, Sastroasmoro S (2012). Effect of 12-week administration of 20-mg long-acting methylphenidate on $\mathrm{Glu} / \mathrm{Cr}$, NAA/Cr, Cho/Cr, and $\mathrm{mI} / \mathrm{Cr}$ ratios in the prefrontal cortices of school-age children in Indonesia: a study using $1 \mathrm{H}$ magnetic resonance spectroscopy (MRS). Clin Neuropharmacol 35: $81-85$.

Wong CG, Stevens MC (2012). The effects of stimulant medication on working memory functional connectivity in attention-deficit/ hyperactivity disorder. Biol Psychiatry 71: 458-466.

Woolrich MW, Jbabdi S, Patenaude B, Chappell M, Makni S, Behrens T et al (2009). Bayesian analysis of neuroimaging data in FSL. Neuroimage 45(1 Suppl): S173-S186.

Supplementary Information accompanies the paper on the Neuropsychopharmacology website (http://www.nature.com/npp) 